\title{
New Virtual Porous Carbons Based on Carbon EDIP Potential and Monte Carlo Simulations
}

\author{
S. Furmaniak \\ Physicochemistry of Carbon Materials Research Group, Department of Chemistry \\ N. Copernicus University, Gagarin St. 7, 87-100 Torun, Poland \\ E-mail:e-mail: sf@chem.umk.pl
}

Received: 26 July 2012; revised: 20 February 2013; accepted: 20 February 2013; published online: 1 March 2013

\begin{abstract}
Using simple Metropolis Monte Carlo simulations, the series of virtual porous carbons (VPCs) is generated. During the computations, the carbon EDIP potential is employed. Structures in the series have systematically changing porosity due to the differences in the carbon density. The obtained VPCs are similar to the model proposed by Harris et al., but they do not show its main drawback, because they contain curved fullerene-like sheets, which are interconnected and form one three-dimensional structure. The porosity of VPCs is characterised using a simple geometrical method proposed by Bhattacharya and Gubbins. In order to confirm the reality of the obtained new model carbons and their usefulness for modelling of adsorption phenomena, Monte Carlo simulations of argon adsorption on them are performed. The obtained isotherms are analysed using standard adsorption methods like $\alpha_{s}$-plots, adsorption potential distributions curves and Dubinin-Astakhov model. The results reveal a close relationship between the systematic changes in the porosity and the adsorption properties. The observed regularities correspond with experimental observations and theoretical studies.
\end{abstract}

Key words: virtual porous carbons, carbon EDIP potential, computer modelling, Monte Carlo simulations

\section{INTRODUCTION}

The term virtual porous carbon (VPC) was proposed by Biggs and Buts [1]. It is used "to describe computer-based molecular models of nanoporous carbons that go beyond the ubiquitous slit pore model and seek to engage with the geometric, topological and chemical heterogeneity that characterises almost every form of nanoporous carbon" [1]. Different VPC models were proposed [1-3]. The most popular VPC models were proposed by Biggs et al. [1,4-7], Do et al. [8-14], and Harris et al. [15-21]. VPCs are also generated using a combination of experimental diffraction data and Monte Carlo simulations (reverse Monte Carlo (RMC) or hybrid reverse Monte Carlo (HRMC) techniques) - see for example [22-29]. HRMC methods seem to be the most sophisticated way to generate realistic VPCs. However, they have some drawbacks [3]. For the given carbon it is possible to generate an infinitive number of replicas that are not unique. Obtained in this way VPCs models are not "flexible", either, i.e. systematic modification of their properties (like porosity) is practically impossible [3]. Also other simulation methods (based mainly on minimization of carbon structure energy and different force fields) are still used to generate new VPCs - see for example [30-32].

The VPCs based on above mentioned Harris model were successfully used in numerous papers of the author and the co-workers for modelling the adsorption properties of activated carbons [3, 33-43]. Harris et al. [15-21] proposed their model basing on the studies of typical non-graphitizing microporous carbons using high-resolution transmission electron microscopy (HRTEM). A careful examination of the obtained HRTEM images exhibited the presence of curved carbon sheets (fullerene-like). Therefore, they proposed a new model of nongraphitizing carbon consisting of discrete fragments of curved carbon sheets, in which pentagons and heptagons are dispersed randomly throughout networks of hexagons. The presence of curved fullerene-like fragments explains important properties of this type of activated carbon like their porosity and high resistance to graphitization. The fact that Harris model contains individual unbound carbon fragments (see for example figure 1 in [34]) is its main drawback. In the case of real carbons it 
can be expected that particular fullerene-like fragments are interconnected and form a three-dimensional structure. Despite this failure, the utility of Harris et al. model is unquestionable. It may be treated as a good model approximation of the microstructure of real activated carbons, as proven, for example, by recovering different experimentally observed empirical correlations [36].

Powles et al. [30] showed that thermally stable structures consisting of a disordered arrangement of highly curved sheets (similarly to Harris model) can be generated via molecular dynamics (MD) simulations employing the environmentdependent interaction potential (EDIP) for carbon proposed by Marks [44, 45]. The obtained in this way structures contain folded fullerene-like fragments with different degrees of curvature, but they are interconnected [30, 46]. The employment of EDIP potential during MD simulations is quite sophisticated because it requires the calculation of the derivatives of complex formulas in order to determine the forces. Metropolis Monte Carlo (MMC) simulations [47] are a simpler alternative to MD simulations. They also lead to the energetically stable VPCs, but do not require the calculation of derivatives (they are based only on calculations of the energy values).

In the present paper, the new series of VPCs is generated using simple MMC simulations employing the above mentioned carbon EDIP potential. The systematic changes in the series are caused by the changes in density of carbon atoms in the simulation box in the range $0.5-1.3 \mathrm{~g} / \mathrm{cm}^{3}$. The porosity of VPCs is characterised by a simple geometric method proposed by Bhattacharya and Gubbins (BG) [48]. In order to confirm the usefulness of the obtained structures in the modelling of adsorption phenomena, the hyper parallel tempering Monte Carlo (HPTMC) simulations [49] of argon adsorption are performed. The obtained isotherms are analysed using standard adsorption methods: high-resolution $\alpha_{s}$-plots, adsorption potential distributions (APDs) curves, and fitting by Dubinin-Astakhov model. These methods are widely used for the characterisation of carbon materials porosity. The obtained VPCs will be also used in the future to perform systematic simulation studies on the adsorption of polyatomic gases and their mixtures. The modification of the surface chemistry of the structures via the introduction of surface groups is also planned.

\section{METHODS}

\section{VPCs generating}

The structures of VPCs were modelled via the simple MMC simulation in the canonical ensemble (NVT). Nine different densities of VPC were considered. They were 0.5 , $0.6,0.7,0.8,0.9,1.0,1.1,1.2$, and $1.3 \mathrm{~g} / \mathrm{cm}^{3}$ (the subsequent structures were denoted as dx.x, where $\mathrm{x} . \mathrm{x}$ is the density). For each VPC, the starting configuration of carbon atoms in the simulation box (having size $4.5 \times 4.5 \times 4.5 \mathrm{~nm}$ ) was generated randomly. Periodic boundary conditions were applied in all three directions. 600 cycles were performed during simu- lations for each VPC. During one cycle $100 \times N_{c}$ (where $N_{c}$ is the number of $\mathrm{C}$ atoms in the box) attempts of the change of the system state were performed. The state of the system was changed only by random displacement of a randomly selected $\mathrm{C}$ atom. The probability of the change acceptance $\left(P_{a c c}\right)$ depended only on the change in the total energy of the system $\left(U_{t o t}\right)[50]$ :

$$
P_{a c c}=\min \left\{1 ; \exp \left[-\frac{\Delta U_{t o t}}{k_{B} T}\right]\right\}
$$

where $k_{B}$ and $T$ denote the Boltzmann constant and the absolute temperature, respectively. The energy of the system was calculated using the above mentioned carbon EDIP potential $[44,45]$. The temperature was changed during the simulation (see figure 1). It was equal to $4500 \mathrm{~K}$ for the first 100 cycles. Then it was linearly reduced to $100 \mathrm{~K}$ during 400 cycles and, finally, the last 100 cycles were performed for a constant temperature equal to $100 \mathrm{~K}$.

The generated in such a way VPCs contained usually a small number of artificial 3-member rings. These rings were usually not connected with the rest of carbonaceous structure or bound with the edges of graphene sheets. The atoms of 3-member rings were removed and the remaining structures were relaxed. In order to relax the VPCs, the energy of the remaining structures was minimised during the above described MMC simulation but starting with the temperature equal to $1000 \mathrm{~K}$. In order to avoid the decrease in density (caused by removing $\mathrm{C}$ atoms) the calculated (from the density) initial number of atoms in each case was increased by 30 extra-atoms. This number was the same for all the structures to simplify the calculations and it was associated with the estimated maximum number of 3-member rings. The assumed number of the extra-atoms was close to the number of atoms which were removed in fact (i.e. between 15 and 44 C atoms).

\section{Porosity of VPCs}

In order to characterise the porosity of the obtained VPCs the above mentioned simple geometrical BG method was used [48]. During the calculation, for each structure the uniform grid $(100 \times 100 \times 100)$ of points was generated in the box. For each point (located in a pore), the largest sphere containing this point and situated in the pore was found in an iterative way (for details see for example [37, 46]). Its diameter corresponds with the size of the pore containing the point. The collection of the data for all the points makes it possible to determine the histogram of pore sizes (i.e. the probabilities of finding the pores having the given effective diameter $\left.\left(d_{e f f}\right)-P\left(d_{e f f}\right)\right)$. The integral curves connected with the histograms were also calculated [46]:

$$
P_{i n t}\left(d_{e f f}\right)=\sum_{d \leq d_{e f f}} P(d)
$$

The $P_{\text {int }}\left(d_{\text {eff }}\right)$ value provides the information what per- 
centage of pores has a diameter not larger than $d_{\text {eff }}$. The obtained histograms were also used to calculate the average size of the pores accessible for argon atoms $\left(d_{e f f, a c c, a v}\right)$ [46]:

$$
d_{e f f, a c c, a v}=\frac{\sum_{d_{e f f} \geq 0.3405 \mathrm{~nm}} d_{e f f} \times P\left(d_{e f f}\right)}{\sum_{d_{e f f} \geq 0.3405 \mathrm{~nm}} P\left(d_{e f f}\right)} .
$$

The used lower limit of pore size $(0.3405 \mathrm{~nm})$ is the diameter of Ar atom (the collision diameter - see below). For the obtained VPCs the ring statistics were also computed using the shortest-path algorithm of Franzblau [51]. During the calculations, bonds were determined by a cut-off distance of $0.185 \mathrm{~nm}$ [46] and the rings up to 8-member ones were considered.

\section{Simulations of argon adsorption isotherms}

Argon adsorption isotherms on generated VPCs were simulated using the above mentioned HPTMC method [49]. The simulations were performed for Ar boiling temperature $(T=87 \mathrm{~K})$. For each system, 142 replicas (corresponding to the relative pressures from $1.0 \times 10^{-10}$ to 1.0 ) were considered. The HPTMC simulations utilized $1 \times 10^{7}$ cycles (one cycle $=100$ attempts of the change of each replica state by (i) creation, (ii) annihilation or (iii) displacement of a randomly chosen atom with equal probabilities, and one attempt of a configuration swap between a pair of randomly chosen replicas). The first $2 \times 10^{6}$ cycles were discarded to guarantee equilibration.

Each argon atom and each carbon atom building the VPCs were modelled as a simple Lennard-Jones (LJ) centre. The truncated LJ potential was used [50]. The following values of LJ parameters (i.e. the collision diameter $(\sigma)$ and the potential well depth $(\varepsilon)$ ) were used: (i) for Ar atoms: $\sigma_{f f}=0.3405 \mathrm{~nm}, \varepsilon_{f f} / k_{B}=119.8 \mathrm{~K}$ [52] and (ii) for $\mathrm{C}$ atoms: $\sigma_{s s}=0.34 \mathrm{~nm}, \varepsilon_{s s} / k_{B}=28.0 \mathrm{~K}$ [53]. Cross-interaction parameters were calculated using Lorentz-Berthelot mixing rules [50]. For each type of interactions, the cut-off distance was assumed as equal to $5.0 \times \sigma_{i j}$.

The average number of Ar atoms in each replica $(\langle N\rangle)$ corresponds to the adsorption amount. It makes it possible to calculate the absolute adsorption value per unit of carbon mass [46]:

$$
a=\frac{\langle N\rangle}{N_{C} \times M_{C}}
$$

where $N_{C}$ and $M_{C}$ are the number of carbon atoms in the box and the molar mass of carbon, respectively. In order to illustrate the degree of filling of pores accessible for the adsorbate, the argon density in pores was also calculated:

$$
\rho=\frac{\langle N\rangle}{N_{A v} \times V_{a c c}}
$$

where $N_{A v}$ is the Avogadro number and $V_{a c c}$ is the volume of pores accessible for Ar atoms. $V_{a c c}$ was computed using
Monte Carlo integration. The size of pores (limiting their accessibility) was determined analogically as in the above described BG method. The isosteric enthalpies of adsorption were calculated from the theory of fluctuations [50]:

$$
q^{s t}=R T-\frac{\langle U N\rangle-\langle U\rangle\langle N\rangle}{\left\langle N^{2}\right\rangle-\langle N\rangle^{2}}
$$

where $R$ is the universal gas constant, $\langle\ldots\rangle$ is the ensemble average and $U$ is the configuration energy of the system.

\section{Analysing adsorption isotherms}

The high-resolution $\alpha_{s}$-plots method [54] was the first technique used for analysing the obtained Ar adsorption isotherms. $\alpha_{s}$-plot presents the values of adsorption on the studied material as a function of the normalised adsorption on a reference material [54]. The reference material should have the same chemical nature as the studied one and should be nonporous. In the case of adsorption on real carbonaceous materials, carbon black is usually used as the reference, but in the case of the simulated isotherms the reference isotherm is very often obtained also via the simulation [46, 54-58]. In this work, the reference Ar adsorption isotherm was simulated (using the above described HPTMC method) for an ideal slit-like pore having an effective diameter equal to $20.0 \mathrm{~nm}$.

The obtained isotherms were also converted into APDs curves [59-61]. The APDs curve is the first derivative of the so called characteristic curve, which presents adsorption as a function of the adsorption potential $\left(A_{p o t}\right)$ defined as:

$$
A_{\text {pot }}=R T \times \ln \frac{p_{s}}{p}
$$

where $p$ and $p_{s}$ are equilibrium and saturated pressure of adsorptive. The differentiation was performed numerically by the approximation of the data by the empirical function (the results were calculated with the resolution of $0.01 \mathrm{~kJ} / \mathrm{mol}$ ).

Finally, the simulated isotherms were fitted by the Dubinin-Astakhov (DA) model, which is one of the most popular equations used in the adsorption area $[36,62,63]$. The DA adsorption isotherm equation may be written as [46]:

$$
a=a_{0} \times \exp \left[-\left(\frac{A_{p o t}}{\beta E_{0}}\right)^{n}\right]
$$

where $a_{0}$ is the maximum adsorption value, $A_{\text {pot }}$ is defined by equation (7), $\beta$ is the affinity coefficient (for $\operatorname{Ar} \beta=0.31$ [64]), $E_{0}$ is the characteristic energy of adsorption, and $n$ is the parameter related to the material heterogeneity. The isotherms were described by DA model in the range of the relative pressure up to 0.1 (this range is also widely used in the case of fitting the real adsorption isotherms). During the fitting, the genetic algorithm proposed by Storn and Price [65] was employed (this method was previously successfully used for fitting different data sets [66-73]). The goodness 
of the fit was estimated using the determination coefficient $(D C)$ defined as [46]:

$$
D C=1-\frac{\sum_{i}\left(a_{\text {theo }, i}-a_{\text {sim }, i}\right)^{2}}{\sum_{i}\left(a_{s i m, i}-\bar{a}_{s i m}\right)^{2}}
$$

where $a_{\text {theo }, i}$ and $a_{\text {sim }, i}$ are the predicted by DA model and the simulated adsorption amount for $i$-th point, and $\bar{a}_{\text {sim }}$ is the average value of simulated adsorption.

\section{RESULTS AND DISCUSSION}

Figure 1 presents the changes in the total energy of the system during MMC simulation for the d1.0 structure (similar regularities are also observed for the other systems). One can see that the high energy of the initial randomly-generated configuration is gradually minimised. The initial reduction in the total energy value (for $T=4500 \mathrm{~K}$ ) is connected with the creation of bonds network among carbon atoms. The further stabilization takes place with a decrease in temperature. The changes in the total energy decrease with the temperature reduction. Simultaneously, the percentage of accepted attempts of atoms displacement also decreases (not shown). For the last 100 cycles $(T=100 K)$, it is less than $0.1 \%$ and the energy reaches the stable final value. Figure 2 presents schematically structures of the obtained VPCs (it may seem that there are some single atoms or unbound several-atoms fragments, but this is only an illusion, because the bonds formed via the periodic boundary conditions are not shown). The presented structures contain folded graphene sheets with a different degree of curvature. They are similar to above mentioned Harris model, but they do not have its main drawback - the sheets are interconnected and they form one three-dimensional structure.

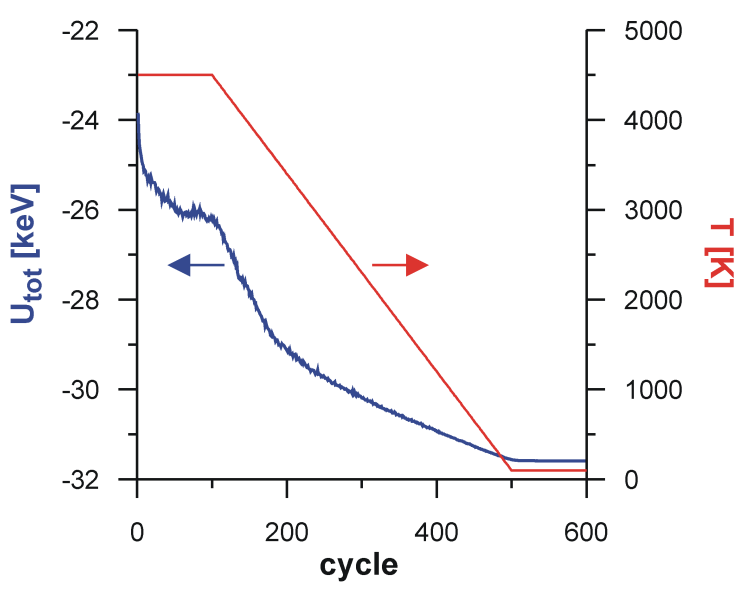

Fig. 1. The changes in total energy and temperature during the MMC simulation for the d1.0 structure

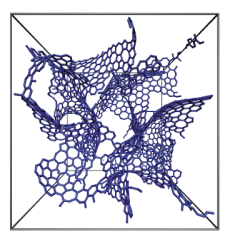

d0.5

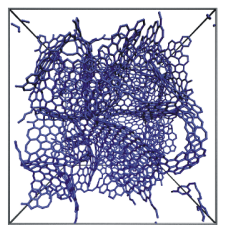

d0.8

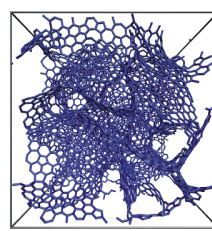

d1.1

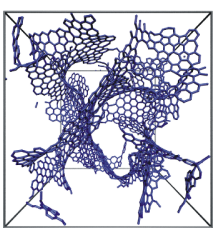

d0.6

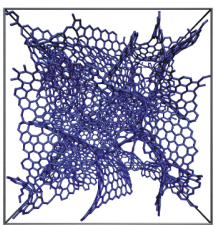

d0.9

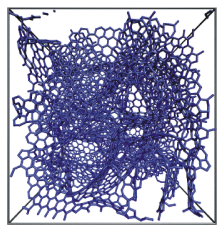

d1.2

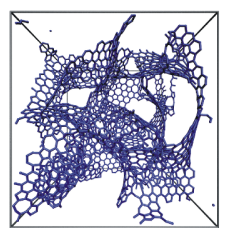

d0.7

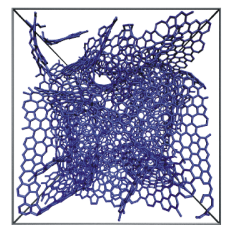

d1.0

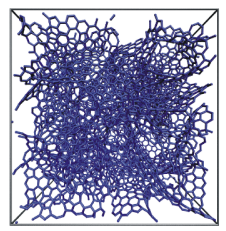

d1.3
Fig. 2. Schematic representation of the obtained VPCs (the frames reflect the size of the simulation box)

Table 1 collects the basic parameters of the obtained VPCs. The ring statistics show that the 6-member rings in structures are dominant for all the VPCs. There are also 5member, 7-member, and 8-member rings. The presence of these rings (especially of the first two kinds) is responsible for the observed curvature of the carbon sheets. The 4-member rings appearing in the case of some structures are usually formed at the connection of fragments of different sheets. Obviously, the volume of pores accessible for Ar atoms decreases with the rise in the number of carbon atoms in the box.

Figure 3 presents the histograms of pore diameters obtained from BG method for all the considered VPCs. All the structures are strictly microporous (i.e. pore sizes do not exceed $2 \mathrm{~nm}$ ). One can also see that the change in the carbon density in the box causes systematic changes in the porosity. Generally, the rise in the density is connected with wider pores vanishing. The d1.1 structure is the only exception. In this case, a certain percentage of the bigger pores (ca. $1.6 \mathrm{~nm}$ ) is also present. This fact may be caused by the non-uniform distribution of carbon atoms in the randomly-generated initial configuration. Nevertheless, the average pore size decreases from the d0.5 structure up to the d1.3 one (see table 1). The last two VPCs (i.e. d1.2 and d1.3) have only quite small pores up to ca. $1 \mathrm{~nm}$. In addition, the rise in the density of carbon atoms in the box is connected with the increase in the percentage of the smallest pores which are inaccessible for $\mathrm{Ar}$ atoms. 
Tab. 1. Basic characteristics of the obtained VPCs

\begin{tabular}{|c|c|c|c|c|c|c|c|c|c|}
\hline \multirow{3}{*}{ VPC } & \multirow{3}{*}{$\begin{array}{l}\text { Number of car- } \\
\text { bon atoms }\end{array}$} & \multirow{3}{*}{$\begin{array}{l}\text { Average diameter of } \\
\text { pores accessible for } \mathrm{Ar} \\
\text { atoms } d_{e f f, a c c, a v}\end{array}$} & \multirow{2}{*}{\multicolumn{2}{|c|}{$\begin{array}{c}\text { Volume of pores } \\
\text { accessible for Ar atoms } V_{a c c}\end{array}$}} & \multicolumn{5}{|c|}{ Number of N-member rings } \\
\hline & & & & & \multicolumn{5}{|c|}{$N$} \\
\hline & & & {$\left[\mathrm{nm}^{3} / \mathrm{box}\right]$} & {$\left[\mathrm{cm}^{3} / \mathrm{g}\right]$} & 4 & 5 & 6 & 7 & 8 \\
\hline $\mathrm{d} 0.5$ & 2262 & 1.224 & 69.86 & 1.548 & 1 & 222 & 495 & 162 & 35 \\
\hline d0.6 & 2737 & 1.125 & 65.37 & 1.198 & 1 & 234 & 682 & 200 & 35 \\
\hline d0.7 & 3192 & 1.053 & 60.82 & 0.955 & 0 & 336 & 458 & 261 & 51 \\
\hline $\mathrm{d} 0.8$ & 3658 & 1.002 & 56.25 & 0.771 & 0 & 378 & 928 & 286 & 73 \\
\hline d0.9 & 4119 & 0.907 & 51.91 & 0.632 & 1 & 409 & 1087 & 335 & 76 \\
\hline $\mathrm{d} 1.0$ & 4573 & 0.789 & 47.05 & 0.516 & 1 & 477 & 1219 & 395 & 85 \\
\hline $\mathrm{d} 1.1$ & 5035 & 0.787 & 42.39 & 0.422 & 0 & 531 & 1384 & 418 & 115 \\
\hline $\mathrm{d} 1.2$ & 5492 & 0.672 & 36.66 & 0.335 & 1 & 613 & 1509 & 467 & 125 \\
\hline $\mathrm{d} 1.3$ & 5949 & 0.670 & 32.18 & 0.271 & 1 & 641 & 1704 & 511 & 148 \\
\hline
\end{tabular}
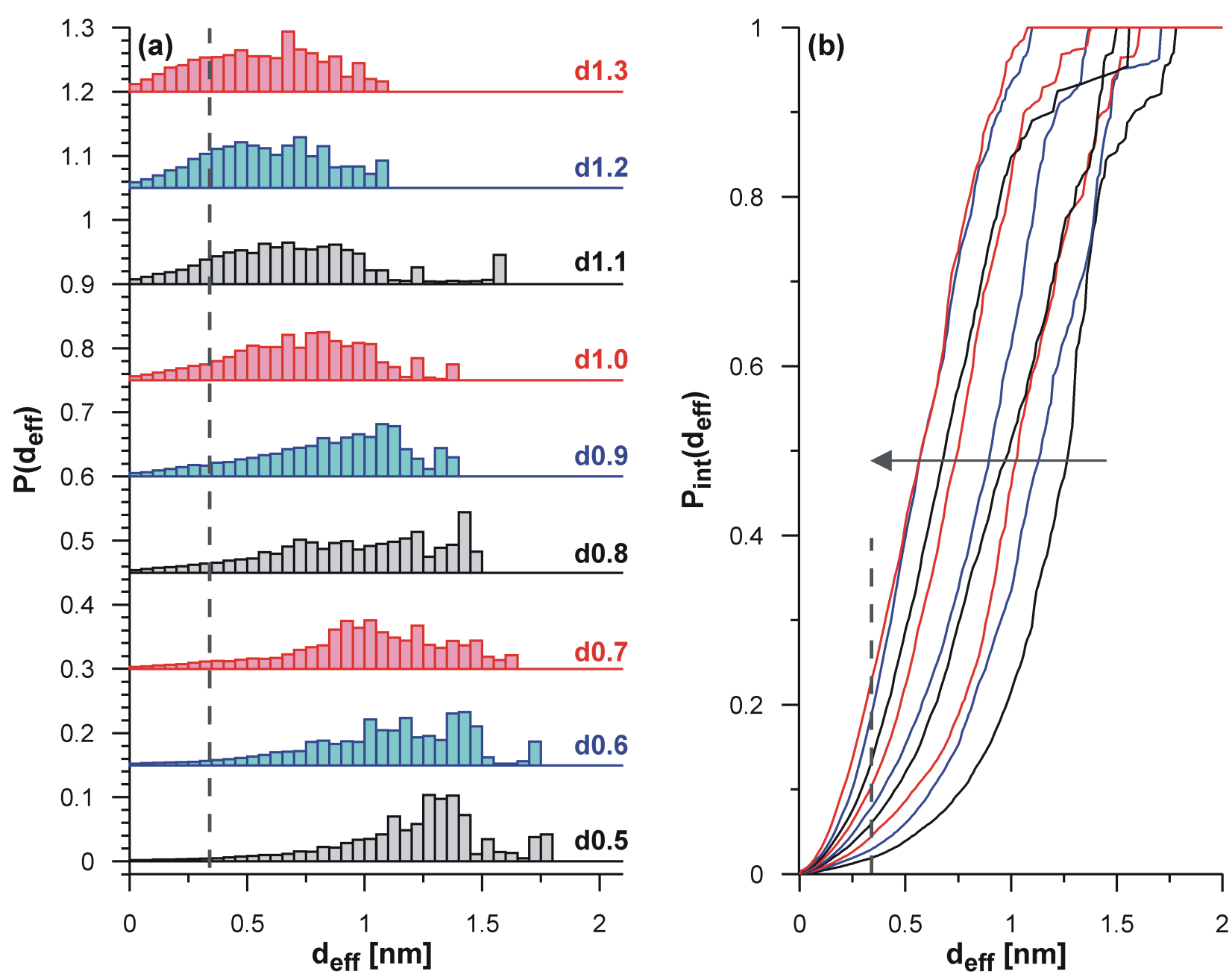

Fig. 3. (a) Histograms of pore diameters for the considered VPCs obtained from BG method (the dashed line represents the size of Ar atoms, subsequent histograms were shifted by 0.15 from the previous ones). (b) Integral curves related to the histograms (the arrow shows the direction of changes from the $\mathrm{d} 0.5$ structure up to the $\mathrm{d} 1.3$ one) 
Figure 4 presents the comparison of the simulated $\mathrm{Ar}$ isotherms and the related isosteric enthalpy of adsorption for all the considered VPCs. In addition, figure 5 presents the snapshots of equilibrium configuration of Ar atom for the selected structures and relative pressures. The observed changes in the shapes of isotherms (from the d0.5 structure up to the $\mathrm{d} 1.3$ one) reflect the differences in the properties of VPCs. The maximal number of Ar atoms in the box and the adsorption capacity (per unit of carbon mass) decrease with the rise of the carbon density. This is caused by the decrease in the volume of accessible pores. The decrease in the adsorption capacity is higher, because it is also affected by the rise in the number of carbon atoms in the box (see equation (4)). However, the maximum density of $\mathrm{Ar}$ in pores (figure $4 \mathrm{c}$ ) is similar for all the structures and close to the density of liquid $\mathrm{Ar}\left(35 \mathrm{~mol} / \mathrm{dm}^{3}\right)$ but below the density of solid $\mathrm{Ar}\left(40.5 \mathrm{~mol} / \mathrm{dm}^{3}\right.$ [74]). Thus, argon atoms for high relative pressures fill the accessible volume of pores. The maximal density is slightly higher for the few last VPCs. This fact may indicate a higher uptake of Ar atoms in the smallest pores.
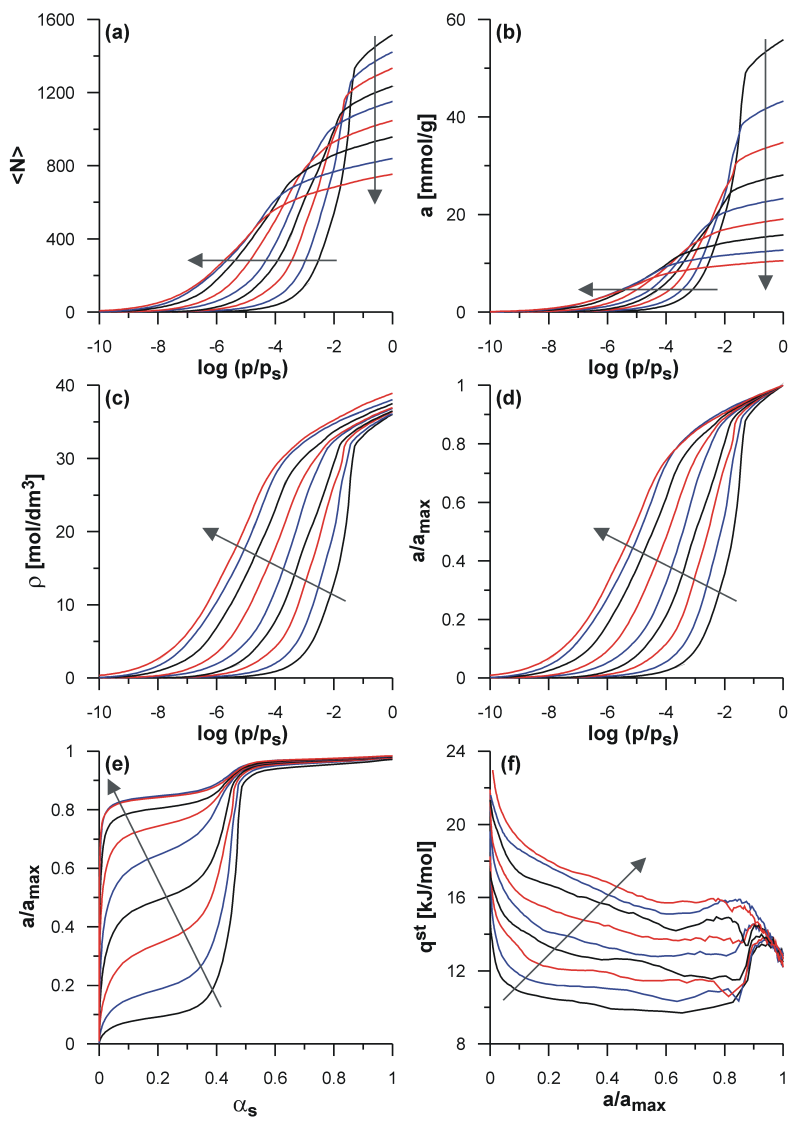

Fig. 4. Comparison of Ar adsorption simulation results $(T=87 \mathrm{~K})$. (a) average number of Ar atoms in the simulation box, (b) adsorption per unit of carbon mass, (c) Ar density in pores, (d) relative adsorption, (e) related $\alpha_{s}$-plots, and (f) related isosteric enthalpy of adsorption. The arrows show the direction of changes from the $\mathrm{d} 0.5$ structure up to the d1.3 one

Also the shape of the isotherms changes regularly from the $\mathrm{d} 0.5$ structure up to the $\mathrm{d} 1.3$ one. The changes are compatible with the differences in the porosity of VPCs. The isotherms become sharper with the decrease in the average pore diameter, i.e. a lower pressure value is necessary to obtain a certain coverage. Differences in the shape are clearly seen especially when the isotherms are presented as the relative adsorption $\left(a / a_{\max }\right)$ - see figure $4 \mathrm{~d}$. These changes are caused by the rise in the adsorption energy with the decrease in the pore size. The reduction of the pressure needed to fill the pore is also confirmed by the snapshots presented in figure 5. For example, in the case of the $\mathrm{d} 0.5$ structure, the pores are not filled for $p / p_{s}=10^{-4}$, while the same pressure is sufficient to almost completely fill pores of the $\mathrm{d} 1.3$ structure. The rise in the adsorption energy for VPCs with the smallest pores is also confirmed by the isosteric enthalpy of adsorption (figure 4f), which increases from the structure d0.5 up to the $\mathrm{d} 1.3$ one. It is worth noticing that similar relations between the shape of isotherms and the porosity are observed experimentally (see for example [59]).

d0.5
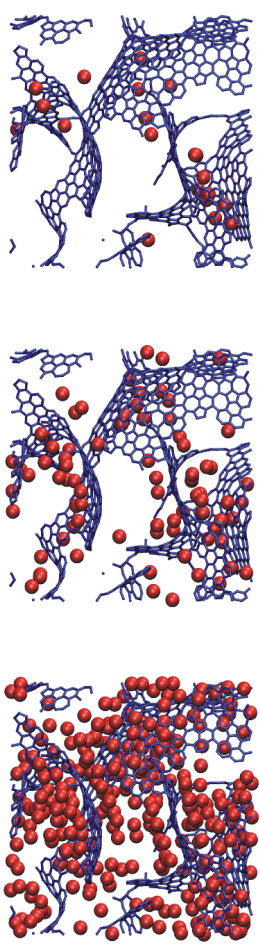

d0.9

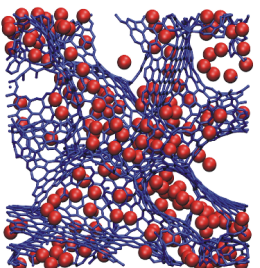

$\mathrm{p} / \mathrm{p}_{\mathrm{s}}=1.0 \times 10^{-4}$

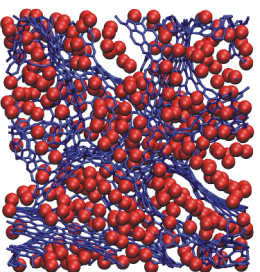

$p / p_{s}=1.0 \times 10^{-3}$

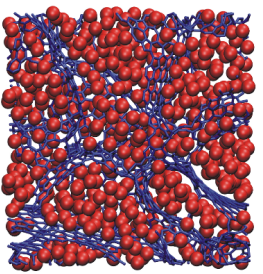

$\mathrm{p} / \mathrm{p}_{\mathrm{s}}=1.0 \times 10^{-2}$ d1.3
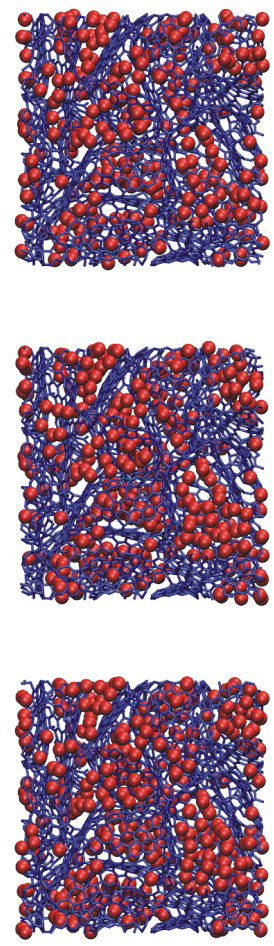

Fig. 5. Selected snapshots of equilibrium configurations for the selected structures (the view after dividing simulation boxes into two equal parts)

Figure 4e collects the $\alpha_{s}$-plots connected with the obtained Ar adsorption isotherms. Both the filling swing (FS) and the condensation swing (CS) [54] are presented for all 
the curves. These deviations from the linearity reflect the increase in the adsorption energy in micropores in comparison with adsorption on the flat surface of the reference material. The FS swing (for small $\alpha_{s}$ values) is connected with the filling of pores having the diameter below $1 \mathrm{~nm}$ [54]. Therefore, in the case of d0.5 structure (having mainly the pores larger than $1 \mathrm{~nm}$ ) this swing is only slightly marked. The FS swing increases passing to the d1.3 structure. The opposite trend is observed in the case of the CS swing which is connected with condensation in the larger micropores [54]. This is caused by the decrease in the percentage of pores above $1 \mathrm{~nm}$. The observed changes correspond with the theoretical results obtained by Setoyama et al. [54] and the results of other simulation studies $[34,46]$.
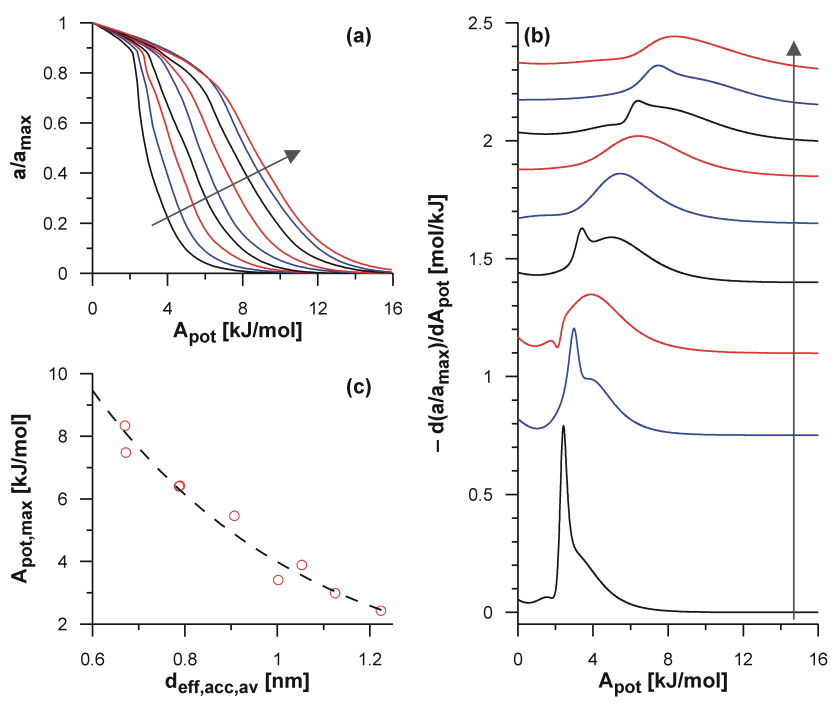

Fig. 6. (a) Characteristic curves connected with isotherms presented in figure 4 (the arrow shows the direction of changes from the $\mathrm{d} 0.5$ structure up to the d1.3 one), (b) comparison of APDs for all the considered systems (subsequent curves were shifted by: 0.0, 0.75, $1.1,1.4,1.65,1.85,2.0,2.15$ and 2.3 , respectively) (c) correlation between the location of maximum on APD curve and the average diameter of pores (the dashed line was drawn to guide the eye)

Figure 6 presents the comparison of the APDs curves related to the simulated Ar isotherms. Also in this case, the relation between porosity and the shape of curves is clearly seen. One can see that the sharp peak observed for the $\mathrm{d} 0.5$ structure (connected with condensation in relatively large micropores of this VPC) becomes smoother and it shifts toward higher values of the adsorption potential for VPCs having smaller pores. This also reflects the changes in the adsorption mechanism connected with the vanishing of the wider micropores. Figure $6 \mathrm{c}$ presents the relation between the location of the maximum on the APD curve $\left(A_{\text {pot, max }}\right)$ and the average pore size. The same hyperbolic-like shape of this relation was reported by Kruk et al. [59] in the case of the experimental data and was confirmed by other simulation studies $[34,46]$.

Tab. 2. Values of the best-fit parameters obtained using DA model (equations (7) and (8)) for the description of the simulated Ar adsorption isotherm in the range $p / p_{s} \leq 0.1$

\begin{tabular}{c|c|c|c|c}
\hline VPC & $\begin{array}{c}a_{0} \\
{[\mathrm{mmol} / \mathrm{g}]}\end{array}$ & $\begin{array}{c}E_{0} \\
{[\mathrm{~kJ} / \mathrm{mol}]}\end{array}$ & $n$ & $D C$ \\
\hline $\mathrm{d} 0.5$ & 85.47 & 8.822 & 1.729 & 0.9943 \\
\hline $\mathrm{d} 0.6$ & 49.15 & 12.70 & 2.325 & 0.9970 \\
\hline $\mathrm{d} 0.7$ & 36.50 & 15.63 & 2.515 & 0.9984 \\
\hline $\mathrm{d} 0.8$ & 28.57 & 18.28 & 2.590 & 0.9992 \\
\hline $\mathrm{d} 0.9$ & 22.88 & 21.19 & 3.044 & 0.9980 \\
\hline $\mathrm{d} 1.0$ & 18.31 & 24.38 & 3.143 & 0.9995 \\
\hline $\mathrm{d} 1.1$ & 15.01 & 27.70 & 3.260 & 0.9993 \\
\hline $\mathrm{d} 1.2$ & 12.10 & 30.36 & 3.447 & 0.9990 \\
\hline $\mathrm{d} 1.3$ & 9.899 & 31.88 & 3.440 & 0.9990 \\
\hline
\end{tabular}
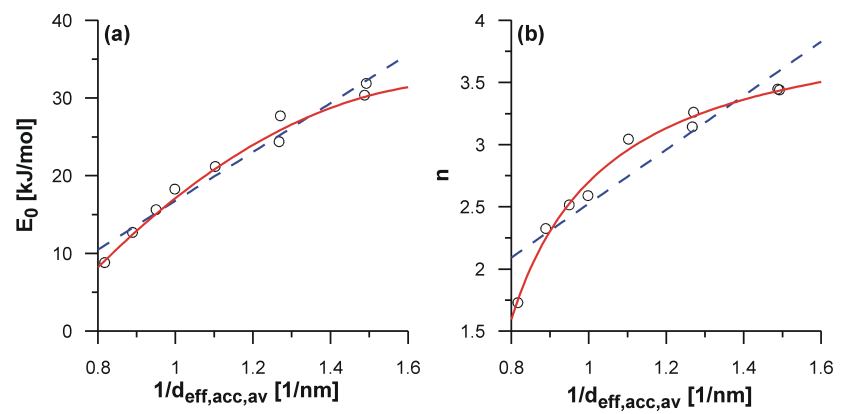

Fig. 7. Correlation between the parameters of the DA model (table 2 ) and the average diameter of pores ((a) characteristic energy, (b) parameter $n$; the lines were drawn to guide the eye)

Table 2 collects the values of the best-fit parameters obtained during the fitting of the simulated isotherms by DA model (equations (7) and (8)) in the range $p / p_{s} \leq 0.1$. The quality of data description is satisfactory as evidenced by the high values of the determination coefficient. The rise in the values of characteristic energy is related to the changes in adsorption energetic caused by the decrease in the average pore diameter. A lot of different mathematical relations connecting a characteristic energy and an average pore size were proposed based on both the experimental measurements and the simulation results - see for example [34] and references therein. The most popular equations assume the inverse proportionality between these two values. As one can see in figure 7, the obtained values of $E_{0}$ may be treated as linearly correlated with $1 / d_{e f f, a c c, a v}$ (the blue dashed line). A similar correlation may be also observed in the case of parameter $n$ in the DA equation (figure $7 \mathrm{~b}$ ). However, a careful analysis of plots $E_{0}=f\left(1 / d_{e f f, a c c, a v}\right)$ and $n=f\left(1 / d_{e f f, a c c, a v}\right)$ 
suggests rather a saturating character of the both relations (see the red lines in figure 7).

\section{CONCLUSIONS}

The proposed method of MMC simulation makes it possible to generate a new series of systematically changing VPCs. The obtained structures are similar to Harris model of the activated carbon microstructure. They contain folded carbon sheets with a different degree of the curvature but they do not show the main drawback of Harris model, because curved fullerene-like fragments are interconnected and form one three-dimensional structure. The usefulness of the obtained VPCs for modelling adsorption phenomena is confirmed by the performed simulation of argon adsorption. The differences in the shape of isotherms reflect the changes in the porosity of VPCs in the series and are analogical to the experimentally observed regularities. The results of the use of a typical adsorption method (like $\alpha_{s}$-plots, APDs curves or DA model) are also intuitively related to the systematic changes in the porosity. They correspond with the results of the experimental observations and theoretical studies. These facts independently confirm the reality of the obtained VPCs.

Since only the adsorption of the simple gas (argon) was studied, further studies seem to be justified. The obtained VPCs will be used to simulate the adsorption of polyatomic gases and their mixtures. The modification of the surface chemistry of the structures via the previously proposed procedure of virtual oxidation [37] is also planned.

\section{Acknowledgments}

The author acknowledges Prof. Nigel A. Marks (Curtin University, Australia) for providing the source code of the EDIP potential, which was very helpful during its implementation in the MMC programme. The author acknowledges the use of the computer cluster at Poznań Supercomputing and Networking Center (Poznań, Poland).

\section{References}

[1] M.J. Biggs and A. Buts, Virtual porous carbons: what they are and what they can be used for, Mol. Simul. 32, 579-593 (2006).

[2] P.A. Gauden, A.P. Terzyk and S. Furmaniak, Modele budowy węla aktywnego wczoraj-dzisiaj-jutro, Wiadomości Chemiczne 62, 403-447 (2008).

[3] A.P. Terzyk, S. Furmaniak, P.A. Gauden, P. J .F. Harris and P. Kowalczyk, Virtual porous carbons, in: J.M.D. Tascón (ed.) Novel Carbon Adsorbents, Elsevier, ch. 3, 2012.
[4] M.J. Biggs, A. Buts and D. Williamson, Molecular simulation evidence for solidlike adsorbate in complex carbonaceous micropore structures, Langmuir 20, 5786-5800 (2004).

[5] M.J. Biggs, A. Buts and D. Williamson, Absolute assessment of adsorption-based porous solid characterization methods: comparison methods, Langmuir 20, 7123-7138 (2004).

[6] Q. Cai, A. Buts, M.J. Biggs and N.A. Seaton, Evaluation of methods for determining the pore size distribution and pore-network connectivity of porous carbons, Langmuir $\mathbf{2 3}$, 8430-8440 (2007).

[7] Q. Cai, A. Buts, N.A. Seaton and M.J. Biggs, A pore network model for diffusion in nanoporous carbons: validation by molecular dynamics simulation, Chem. Eng. Sci. 63, 33193327 (2008).

[8] D.D. Do and H.D. Do, Modeling of adsorption on nongraphitized carbon surface: GCMC simulation studies and comparison with experimental data, J. Phys. Chem. B 110, 1753117538 (2006).

[9] D.D. Do, D. Nicholson and H.D. Do, Heat of adsorption and density distribution in slit pores with defective walls: GCMC simulation studies and comparison with experimental data, Appl. Surf. Sci. 253, 5580-5586 (2007).

[10] G.R. Birkett and D.D. Do, On the physical adsorption of gases on carbon materials from molecular simulation, Adsorption 13, 407-424 (2007).

[11] A. Wongkoblap and D.D. Do, Characterization of Cabot nongraphitized carbon blacks with a defective surface model: adsorption of argon and nitrogen, Carbon 45, 1527-1534 (2007).

[12] L.F. Herrera, D.D. Do and G.R. Birkett, Comparative simulation study of nitrogen and ammonia adsorption on graphitized and nongraphitized carbon blacks. J. Colloid Interface Sci. 320, 415-422 (2008).

[13] G.R. Birkett and D.D. Do: Characteristic heats of adsorption for slit pore and defected pore models, Langmuir $\mathbf{2 4}$, 4853-4856 (2008).

[14] L.F. Herrera and D.D. Do, Effects of surface structure on the molecular projection area. Adsorption of argon and nitrogen onto defective surfaces, Adsorption 15, 240-246 (2009).

[15] P.J.F. Harris and S.C. Tsang: High-resolution electron microscopy studies of non-graphitizing carbons, Philos. Mag. A 76, 667-677 (1997).

[16] P.J.F. Harris, Structure of non-graphitising carbons, Int. Mater. Rev. 42, 206-218 (1997).

[17] P.J.F. Harris, A. Burian and S. Duber, High-resolution electron microscopy of a microporous carbon, Philos. Mag. Lett. 80, 381-386 (2000).

[18] P.J.F. Harris, New perspectives on the structure of graphitic carbons, Crit. Rev. Solid State Mater. Sci. 30, 235-253 (2005).

[19] P.J.F. Harris, Z. Liu and K. Suenaga, Imaging the atomic structure of activated carbon, J. Phys.: Condens. Matt. 20, 362201 (2008).

[20] P.J.F. Harris, Fullerene-related structure of non-graphitizing carbons, in: A.P. Terzyk, P.A. Gauden and P. Kowalczyk (eds.) Carbon Materials - Theory and Practice, Research Signpost, Kerala, India, p. 1-14, 2008.

[21] P.J.F. Harris, Z. Liu and K. Suenaga, Imaging the structure of activated carbon using aberration corrected TEM. J. Phys.: Conf. Ser. 241, 012050 (2010) 
[22] T. Petersen, I. Yarovsky, I. Snook, D.G. McCulloch and G. Opletal, Structural analysis of carbonaceous solids using an adapted reverse Monte Carlo algorithm, Carbon 41, 2403 2411 (2003).

[23] G. Opletal, T.C. Petersen, D.G. McCulloch, I.K. Snook and I. Yarovsky, The structure of disordered carbon solids studied using a hybrid reverse Monte Carlo algorithm, J. Phys.: Condens. Matt. 17, 2605-2616 (2005).

[24] S.K. Jain, R.J.M. Pellenq, J.P. Pikunic and K.E. Gubbins, Molecular modeling of porous carbons using the hybrid reverse Monte Carlo method, Langmuir 22, $9942-9948$ (2006).

[25] S.K. Jain, K.E. Gubbins, R.J.M. Pellenq and J.P. Pikunic, Molecular modeling and adsorption properties of porous carbons, Carbon 44, 2445-2451 (2006).

[26] B. Coasne, S.K. Jain, L. Naamar and K.E. Gubbins, Freezing of argon in ordered and disordered porous carbon, Phys. Rev. B 76, 085416 (2007).

[27] B. Coasne, C. Alba-Simionesco, F. Audonnet, G. Dosseh and K.E. Gubbins, Adsorption, structure and dynamics of benzene in ordered and disordered porous carbons, Phys. Chem. Chem. Phys. 13, 3748-3757 (2011).

[28] J.C. Palmer and K.E. Gubbins, Atomistic models for disordered nanoporous carbons using reactive force fields, Microporous Mesoporous Mater. 154, 24-37 (2012).

[29] P. Kowalczyk, P.A. Gauden and A.P. Terzyk, Structural properties of amorphous diamond-like carbon: percolation, cluster, and pair correlation analysis. RSC Adv. 2, 4292-4298 (2012).

[30] R.C. Powles, N.A. Marks and D.W.M. Lau, Self-assembly of $s p^{2}$-bonded carbon nanostructures from amorphous precursors, Phys. Rev. B 79, 075430 (2009).

[31] A. Kumar, R.F. Lobo and N.J. Wagner, Grand canonical Monte Carlo simulation of adsorption of nitrogen and oxygen in realistic nanoporous carbon models, AIChE J. 57, 1496-1505 (2011).

[32] L.J. Peng and J.R. Morris, Structure and hydrogen adsorption properties of low density nanoporous carbons from simulations, Carbon 50, 1394-1406 (2012).

[33] A.P. Terzyk, S. Furmaniak, P.A. Gauden, P.J.F. Harris, R.P. Wesołowski and P. Kowalczyk, Virtual porous carbon (VPC) models: application in the study of fundamental activated carbon properties by molecular simulations, J.F. Kwiatkowski (ed.) Activated Carbon: Classifications, Properties and Applications, Nova Science Publishers, New York, ch. 8, 2011.

[34] A.P. Terzyk, S. Furmaniak, P.A. Gauden, P.J.F. Harris, J. Włoch and P. Kowalczyk, Hyper-parallel tempering Monte Carlo simulations of Ar adsorption in new models of microporous non-graphitizing activated carbon: effect of microporosity, J. Phys.: Condens. Matt. 19, 406208 (2007).

[35] A.P. Terzyk, S. Furmaniak, P.J.F. Harris, P.A. Gauden, J. Włoch, P. Kowalczyk and G. Rychlicki, GCMC simulations of Ar adsorption in new model of non-graphitizing activated carbon. How realistic is the pore size distribution calculated from adsorption isotherms using standard methods?, Phys. Chem. Chem. Phys. 9, 5919-5929 (2007).

[36] A.P. Terzyk, S. Furmaniak, P.A. Gauden, P.J.F. Harris and J. Włoch, Testing isotherm models and recovering empirical relationships for adsorption in microporous carbons using virtual carbon models and grand canonical Monte Carlo simulations, J. Phys.: Condens. Matt. 20, 385212 (2008).

[37] S. Furmaniak, A.P. Terzyk, P.A. Gauden, P.J.F. Harris and P. Kowalczyk, Can carbon surface oxidation shift the pore size distribution curve calculated from $\mathrm{Ar}, \mathrm{N}_{2}$ and $\mathrm{CO}_{2}$ adsorption isotherms? Simulation results for a realistic carbon model, J. Phys.: Condens. Matt. 21, 315005 (2009).

[38] A.P. Terzyk, P.A. Gauden, S. Furmaniak, R.P. Wesołowski and P.J.F. Harris, Molecular dynamics simulation insight into the mechanism of phenol adsorption at low coverages from aqueous solutions on microporous carbons, Phys. Chem. Chem. Phys. 12, 812-817 (2010).

[39] S. Furmaniak, A.P. Terzyk, P.A. Gauden, P.J.F. Harris and P. Kowalczyk, The influence of carbon surface oxygen groups on Dubinin-Astakhov equation parameters calculated from $\mathrm{CO}_{2}$ adsorption isotherm, J. Phys.: Condens. Matt. 22, 085003 (2010).

[40] P.A. Gauden, A.P. Terzyk, S. Furmaniak, P.J.F. Harris and P. Kowalczyk, BET surface area of carbonaceous adsorbents verification using geometric considerations on virtual porous carbon models, Appl. Surf. Sci. 256, 5204-5209 (2010).

[41] A.P. Terzyk, S. Furmaniak, R.P. Wesołowski, P.A. Gauden and P.J.F. Harris, Methane storage in microporous carbons - effect of porosity and surface chemical composition tested on realistic carbon model, B.B. Saha and K.C. $\mathrm{Ng}$ (eds.) Advances in Adsorption Technology, Nova Science Publishers, New York, ch. 14, 2010.

[42] S. Furmaniak, A.P. Terzyk, P.A. Gauden P., Kowalczyk and P.J.F. Harris, The influence of the carbon surface chemical composition on Dubinin-Astakhov equation parameters calculated from $\mathrm{SF}_{6}$ adsorption data - grand canonical Monte Carlo simulation, J. Phys.: Condens. Matt. 23, 395005 (2011).

[43] P .Kowalczyk, P.A. Gauden, A.P. Terzyk, S. Furmaniak and P.J.F. Harris, Displacement of methane by coadsorbed carbon dioxide is facilitated in narrow carbon nanopores, J. Phys. Chem. C 116, 13640-13649 (2012).

[44] N.A. Marks, Generalizing the environment-dependent interaction potential for carbon, Phys. Rev. B 63, 035401 (2000).

[45] N. Marks: Modelling diamond-like carbon with the environment-dependent interaction potential, J. Phys.: Condens. Matt. 14, 2901-2927 (2002).

[46] S. Furmaniak, A.P. Terzyk, P.A. Gauden, N.A. Marks, R.C. Powles and P. Kowalczyk, Simulating the changes in carbon structure during the burn-off process, J. Colloid Interface Sci. 360, 211-219 (2011).

[47] G.J. Opletal, Structural Simulations using the Hybrid Reverse Monte Carlo Method, Ph.D. thesis, RMIT, Melbourne, Australia 2005.

[48] S. Bhattacharya and K.E. Gubbins, Fast method for computing pore size distributions of model materials, Langmuir 22, 7726-7731 (2006).

[49] Q. Yan and J.J. de Pablo: Hyper-parallel tempering Monte Carlo: application to the Lennard-Jones fluid and the restricted primitive model, J. Chem. Phys. 111, 9509-9516 (1999).

[50] D. Frenkel and B. Smit, Understanding Molecular Simulation, Academic Press, San Diego 1996.

[51] D.S. Franzblau, Computation of ring statistics for network models of solids, Phys. Rev. E 44, 4925-4930 (1991). 
[52] D.D. Do and H.D. Do, Effects of potential models in the vapor-liquid equilibria and adsorption of simple gases on graphitized thermal carbon black. Fluid Phase Equilib. 236, 169-177 (2005).

[53] W.A. Steele: The Interaction of Gases with Solid Surfaces, Pergamon Press, Oxford 1974.

[54] N. Setoyama, T. Suzuki and K. Kaneko, Simulation study on the relationship between a high resolution $\alpha_{s}$-plot and the pore size distribution for activated carbon, Carbon $\mathbf{3 6}$, 1459-1467 (1998).

[55] T. Ohba and K. Kaneko, Internal surface area evaluation of carbon nanotube with GCMC Simulation-assisted $\mathrm{N}_{2}$ adsorption, J. Phys. Chem. B 106, 7171-7176 (2002).

[56] A.P. Terzyk, P.A. Gauden, S. Furmaniak and P. Kowalczyk, Heterogeneity on high-resolution $\alpha_{s}$ plots for carbon nanotubes - GCMC study, Phys. Chem. Chem. Phys. 10, 45514554 (2008).

[57] S. Furmaniak, A.P. Terzyk, P.A. Gauden, R.P. Wesołowski and P. Kowalczyk, $\mathrm{Ar}, \mathrm{CCl}_{4}$, and $\mathrm{C}_{6} \mathrm{H}_{6}$ Adsorption outside and inside of the bundles of multi-walled carbon nanotubes simulation study, Phys. Chem. Chem. Phys. 11, 4982-4995 (2009).

[58] A.P. Terzyk, S. Furmaniak, P.A. Gauden and P. Kowalczyk, Fullerene intercalated graphene nanocontainers - the mechanism of Ar adsorption and the test of high pressure $\mathrm{CH}_{4}$ and $\mathrm{CO}_{2}$ storage capacities, Adsorpt. Sci. Technol. 27, 281-296 (2009).

[59] M. Kruk, M. Jaroniec and K.P. Gadkaree, Determination of the specific surface area and the pore size of microporous carbons from adsorption potential distributions, Langmuir 15, 1442-1448 (1999).

[60] J. Choma and M. Jaroniec: A model-independent analysis of nitrogen adsorption isotherms on oxidized active carbons, Colloids Surf. A 189, 103-111 (2001).

[61] J. Choma and M. Jaroniec: Adsorption potential distributions for silicas and organosilicas, Adsorpt. Sci. Technol. 25 , 573-581 (2007)

[62] F. Stoeckli: Dubinin's theory and its contribution to adsorption science, Russ. Chem. Bull. 50, 2265-2272 (2001).

[63] J. Kadlec, The history and present state of Dubinin's theory of adsorption of vapours and gases on microporous solids, Adsorpt. Sci. Technol. 19, 1-24 (2001).
[64] B. McEnaney, Estimation of the dimensions of micropores in active carbons using the Dubinin-Radushkevich equation, Carbon 25, 69-75 (1987).

[65] R. Storn, K. Price, Differential evolution - a simple and efficient heuristic for global optimization over continuous spaces, J. Glob. Optim. 11, 341-359 (1997).

[66] S. Furmaniak, P.A. Gauden, A.P. Terzyk and G. Rychlicki, Water adsorption on carbons - critical review of the most popular analytical approaches, Adv. Colloid. Interface Sci. 137, 82-143 (2008).

[67] S. Furmaniak, A.P. Terzyk, R. Gołembiewski, P.A. Gauden and L. Czepirski, Searching the most optimal model of water sorption on foodstuffs in the whole range of relative humidity, Food Res. Int. 42, 1203-1214 (2009).

[68] S. Furmaniak, A.P. Terzyk, G. Rychlicki, M. Wiśniewski, P.A Gauden, P. Kowalczyk, K.M. Werengowska and K. Dulska, The system: carbon tetrachloride - closed carbon nanotubes analysed by a combination of molecular simulations, analytical modelling and adsorption calorimetry, J. Colloid Interface Sci. 349, 321-330 (2010).

[69] S. Furmaniak, A.P. Terzyk, P.A. Gauden, P.J.F. Harris, M Wiśniewski and P. Kowalczyk, Simple model of adsorption on external surface of carbon nanotubes - a new analytical approach basing on molecular simulation data, Adsorption 16, 197-213 (2010).

[70] S. Furmaniak, A.P. Terzyk, R. Gołembiewski and P.A. Gauden, Surface area of closed carbon nanotubes determined from room temperature measurements of alcohols adsorption, Chem. Phys. Lett. 499, 141-145 (2010).

[71] J.K. Garbacz, S. Furmaniak, A.P. Terzyk and M. Grabiec New model describing adsorption from liquid binary mixtures of nonelectrolytes with limited and unlimited miscibility of components, J. Colloid Interface Sci. 359, 512-519 (2011).

[72] S. Furmaniak, A.P. Terzyk and P.A. Gauden, Some remarks on the classification of water vapor sorption isotherms and Blahovec and Yanniotis isotherm equation, Drying Technol. 29, 984-991 (2011).

[73] S. Furmaniak, The alternative model of water vapour sorption in porous building materials, Transp. Porous Media 95 , 21-23 (2012).

[74] W. Van Witzenburg: Density of solid argon at the triple point and concentration of vacancies, Phys. Lett. A 25, 293-294 (1967). 


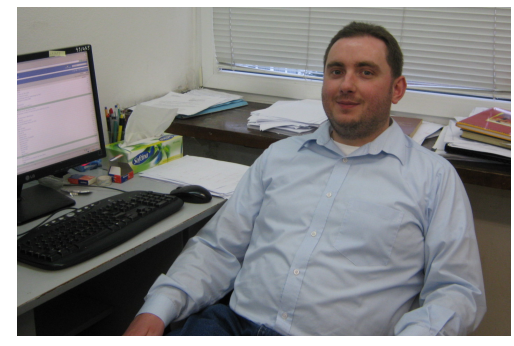

Sylwester Furmaniak was born in Złotów (Poland) in 1979. He studied chemistry at Nicolaus Copernicus University in Torun, where he received his M.Sc. in Physical Chemistry in 2005. He received his Ph.D. degree in Chemistry in 2010 from Nicolaus Copernicus University in Torun (thesis: Sorption Equilibria in Pores of Carbonaceous Materials - Analytical Models and Computer Simulations). He has been a member of "Physicochemistry of Carbon Materials Research Group" at Department of Chemistry, Nicolaus Copernicus University since 2005. His main scientific interests are connected with Monte Carlo simulations of adsorption phenomena and mathematical modelling of adsorption isotherms and related energetic of the process. He is a co-author of more than 60 original papers (excluding conference materials). In 2009 and 2010 he was awarded with the Scholarship for Young Scientists of Foundation for Polish Science. 